\title{
Macular Thickness and Volume Assessed by Spectral-Domain Optical Coherence Tomography in Patients With Coronavirus Disease 2019: A Case- Control Study
}

Mojtaba Abrishami ( $\nabla$ mojtaba_abrishami@yahoo.com )

Mashhad University of Medical Sciences https://orcid.org/0000-0003-2001-7929

Fariba Tohidinezhad

Mashhad University of Medical Sciences

Zahra Emamverdian

Mashhad University of Medical Sciences

Ramin Daneshvar

Mashhad University of Medical Sciences

Neda Saeedian

Mashhad University of Medical Sciences

Mohammad-Reza Ansari-Astaneh

Mashhad University of Medical Sciences

Maria Cristina Savastano

IRCCS: IRCCS Ospedale San Raffaele

\section{Saeid Eslami}

Mashhad University of Medical Sciences

\section{Original research}

Keywords: Coronavirus Disease 2019 (COVID-19), Severe Acute Respiratory Syndrome Corona Virus 2 (SARS-CoV-2), Spectral-Domain Optical Coherence Tomography (SD-OCT), Macula, Retina

Posted Date: December 28th, 2020

DOl: https://doi.org/10.21203/rs.3.rs-133611/v1

License: (1) This work is licensed under a Creative Commons Attribution 4.0 International License. Read Full License 


\section{Abstract}

Purpose: To quantify the retinal thickness and volume using spectral-domain optical coherence tomography (SD-OCT) analysis in the macular region of patients with Coronavirus Disease 2019 (COVID19).

Methods: In a comparative cross-sectional, observational study, patients recovered from COVID-19 were included. All included subjects had a reverse transcription-polymerase chain reaction (RT-PCR) confirmed diagnosis of COVID-19. Macular SD-OCT was performed at least two weeks after recovery from systemic COVID-19. Inner, outer and full retinal thicknesses and volumes were measured in COVID19 recovered patients versus age-matched normal controls.

Results: Twenty-five patients (11 male) with a mean age of $36.4 \pm 11$ years and 60 healthy controls (31 males) with a mean age of $39.3 \pm 7.7$ years were enrolled in the study. There was no statistically significant difference in the retinal thickness or volume measures between the two groups. However, the thickness in the case group was minimally more than the controls.

Conclusion: Retinal thickness in COVID-19 patients may be higher than healthy subjects. Comprehensive ocular examination with special focus on posterior segment manifestations should be considered in these patients.

\section{Introduction}

Severe Acute Respiratory Syndrome Coronavirus (SARS-CoV-2) is a highly contagious infection with multi-organ involvement. Neurological manifestations have been reported throughout the disease course. Therefore, the potential involvement of the central nervous system (CNS) in Coronavirus Disease 2019 (COVID-19) has been proposed (1). Since SARS-CoV-2 enters human cells by binding to the AngiotensinConverting Enzyme (ACE) 2 receptor, cells presenting ACE2 are vulnerable to SARS-CoV-2 infection (2). In addition to the lung alveolar cells, neurons, and glial cells in the CNS(1), several retinal components, including Müller cells, ganglion cells, retinal vascular endothelial cells, and photoreceptor cells, also present the ACE2 receptors(3). Hence, it seems reasonable to expect both ocular and CNS manifestations in the SARS-CoV-2 infection.

Spectral-Domain Optical Coherence Tomography (SD-OCT) is a non-invasive imaging modality with the ability of high resolution, cross-sectional evaluation of the retina. Marinho et al. presented SD-OCT findings in twelve COVID-19 patients and reported hyperreflective retinal lesions in all cases (4), but these findings were questioned (5). In another report, the SARS-CoV-2 RNA was detected in autopsy specimens of patients deceased with COVID-19 (6).

Since the neurological manifestations of the SARS-CoV-2 have not been adequately studied yet, the casualty or coincidence of these findings with COVID-19 in not clear. Usually, critically ill patients have shown a greater risk of developing neurological symptoms $(7,8)$. As the retina and optic nerve are 
extensions of the central nervous system, evaluation of the retinal changes may help to identify the CNS changes as well. Indeed, it has been demonstrated that the SD-OCT is an effective diagnostic modality to detect many CNS pathologies $(9,10)$.

The most common and easily identifiable ocular manifestations of the SARS-CoV-2 have been reported in the ocular surface (e.g. conjunctival hyperemia, chemosis, and epiphora) (11-14). However, there is a paucity of data on other ocular findings in these patients. In this study, we aimed to evaluate the total, inner, and outer retinal thickness and retinal volume in the macula area of a cohort of patients with a confirmed diagnosis of COVID-19 and compare them with a control group.

\section{Methods}

\section{Study Participants}

A comparative observational study was conducted in Imam Reza Hospital, the referral center devoted to COVID-19 patients in Northeast of Iran. All included patients had a definite diagnosis of COVID-19, confirmed by a positive real-time, Reverse Transcription-Polymerase Chain Reaction (RT-PCR) test result of nasopharyngeal swab sample and had at least a two-week recovery period. A control group was retrospectively selected from participants in the ongoing PERSIAN cohort study in the Mashhad University of Medical Sciences (15). The individuals in the control group were examined before January 2020, one month before the first report of a confirmed case of COVID-19 in the region. Two ophthalmologists (MA, MRA) overviewed the charts and SD-OCT images of the control group to ensure there were no abnormalities. Patients with a past medical history of diabetes mellitus, systemic hypertension, or known vascular or neurologic disorders, and those with a history of glaucoma or any intraocular surgeries were excluded from the study in both groups.

\section{Image Acquisition}

Macular area of the eligible subjects was imaged using an SD-OCT machine (AngioVue, Optovue RTVue XR Avanti, Optovue, Fremont, CA). Raster and Retina Map protocols of the device software were used to evaluate the following retinal thicknesses in the macular area: Full retina (internal limiting membrane (ILM) to Bruch membrane (BRM)), inner retina (ILM to the interface of the inner plexiform layer (IPL) and the inner nuclear layer (INL)), and outer retina (interface of the IPL and INL to BRM). Two experienced retina specialists (MA and MRA) evaluated the accuracy of SD-OCT image segmentation and corrected them if needed. All subjects in both groups were examined using the same machine. All images with Signal Strength Index (SSI) of less than 50 were excluded. Between both eyes, SD-OCT image with better SSI was included.

\section{Statistical Analysis}

Statistical analysis was performed using the SPSS program for Windows, version 20 (IBM SPSS Statistics, IBM Corporation, Chicago, IL, USA). Descriptive statistics were used to describe the variables by providing central tendency measures for continuous variables and frequencies for categorical variables. 
The normal distribution of variables was evaluated through the Shapiro-Wilk test and normality plots. Based on the distribution, the independent-samples $\mathrm{T}$ test or Mann-Whitney $\mathrm{U}$ test was used to compare thickness measurements between the independent samples (control and case groups). For all tests, a pvalue less than or equal to 0.05 was considered as statistically significant.

\section{Ethical Considerations}

The study protocol adhered to the tenets of the 1964 Declaration of Helsinki and its later amendment. All subjects participated in the study voluntarily at their convenience and provided written informed consent at the enrolment. The study protocol was approved by the Medical Ethics Committee at the Mashhad University of Medical Sciences, Mashhad, Iran (IR.MUMS.REC.1399.104).

\section{Results}

Twenty-five patients (11 male) with a mean age of $36.4 \pm 11$ years and 60 healthy controls (31 male) with a mean age of $39.3 \pm 7.7$ years were enrolled in the study. Age $(P=0.263)$ and gender $(P=0.635)$ were not significantly different between the two groups. Mean SSI was $75.7 \pm 6.8$ in the COVID-19 cases and 72.3 \pm 8.3 in the normal controls $(P=0.181)$.

As shown in Table 1, the full retinal thickness of the case and control groups in parafoveal $(311.2 \pm 19.0$ vs. $308.9 \pm 14.1$, respectively; $P=0.606)$ and perifoveal $(285.4 \pm 16.3$ vs. $282.7 \pm 10.1$, respectively; $P=$ 0.142 ) rings were not statistically significant different. As listed in Table 2 and Table 3 , the unremarkable increase was also observed in the inner (parafovea: $129.1 \pm 9.6$ vs. $128.1 \pm 7.4, P=0.629$; perifovea: $113.9 \pm 8.1$ vs. $112.7 \pm 5.3, P=0.200$ ) and outer (parafovea: $181.9 \pm 10.5$ vs. $180.7 \pm 8.7, P=0.559$; perifovea: $171.6 \pm 8.9$ vs. $170.1 \pm 6.7, \mathrm{P}=0.175$ ) retinal thicknesses. It is noteworthy that the case group had also no statistically significant difference in foveal thickness compared to the control group in inner, outer or full retinal thickness analysis. Moreover, retinal volumes are also unremarkably increased in different layers of retina (Tables 1-3).

Table 1: Comparison of the Full Retinal Thickness between the COVID-19 Patients ( $N=25)$ and Control Group $(\mathrm{N}=60)$ 


\begin{tabular}{|lllllll|}
\hline Variable & \multicolumn{2}{l}{ Retinal Thickness } & \multicolumn{3}{l|}{ Retinal Volume } \\
\cline { 2 - 7 } & Control & Case & P-value & Control & Case & P-value \\
\hline Fovea & $245.7 \pm 20.0$ & $248.3 \pm 20.5$ & 0.407 & $0.193 \pm 0.016$ & $0.195 \pm 0.016$ & 0.415 \\
\hline Parafovea & $308.9 \pm 14.1$ & $311.2 \pm 19.0$ & 0.606 & $1.940 \pm 0.088$ & $1.954 \pm 0.119$ & 0.633 \\
\hline S Hemisphere & $308.7 \pm 13.7$ & $312.2 \pm 18.6$ & 0.417 & $0.970 \pm 0.043$ & $0.981 \pm 0.058$ & 0.423 \\
\hline I Hemisphere & $308.9 \pm 14.9$ & $310.1 \pm 19.5$ & 0.794 & $0.971 \pm 0.047$ & $0.974 \pm 0.061$ & 0.813 \\
\hline Temporal & $299.5 \pm 14.6$ & $303.8 \pm 19.2$ & 0.265 & $0.470 \pm 0.023$ & $0.485 \pm 0.045$ & 0.137 \\
\hline Superior & $312.6 \pm 13.0$ & $315.3 \pm 18.4$ & 0.46 & $0.491 \pm 0.020$ & $0.495 \pm 0.029$ & 0.429 \\
\hline Nasal & $311.7 \pm 16.5$ & $314.1 \pm 19.2$ & 0.654 & $0.490 \pm 0.026$ & $0.493 \pm 0.030$ & 0.63 \\
\hline Inferior & $311.6 \pm 14.7$ & $311.2 \pm 20.5$ & 0.965 & $0.489 \pm 0.023$ & $0.489 \pm 0.032$ & 0.923 \\
\hline Perifovea & $282.7 \pm 10.1$ & $285.4 \pm 16.3$ & 0.142 & $3.552 \pm 0.127$ & $3.587 \pm 0.204$ & 0.147 \\
\hline S Hemisphere & $284.6 \pm 10.1$ & $287.9 \pm 16.2$ & 0.124 & $1.788 \pm 0.064$ & $1.809 \pm 0.101$ & 0.117 \\
\hline I Hemisphere & $280.8 \pm 10.8$ & $283.0 \pm 16.9$ & 0.257 & $1.764 \pm 0.068$ & $1.778 \pm 0.106$ & 0.267 \\
\hline Temporal & $271.9 \pm 11.1$ & $276.0 \pm 15.4$ & 0.077 & $0.853 \pm 0.035$ & $0.867 \pm 0.048$ & 0.056 \\
\hline Superior & $284.3 \pm 10.4$ & $287.8 \pm 17.1$ & 0.089 & $0.893 \pm 0.033$ & $0.904 \pm 0.053$ & 0.093 \\
\hline Nasal & $298.1 \pm 11.7$ & $299.9 \pm 17.7$ & 0.518 & $0.936 \pm 0.037$ & $0.942 \pm 0.055$ & 0.475 \\
\hline Inferior & $276.4 \pm 11.2$ & $278.1 \pm 17.3$ & 0.377 & $0.868 \pm 0.035$ & $0.874 \pm 0.054$ & 0.357 \\
\hline
\end{tabular}

Notes: The unit of measurement thicknesses and volumes were $\mu \mathrm{m}$ and $\mathrm{mm}^{3}$, respectively. Values represented as mean \pm SD and analysis made by Mann-Whitney $U$ test.

Abbreviations: S, superior; I, inferior

Table 2: Comparison of the Inner Retinal Thickness between the COVID-19 Patients ( $N=25)$ and Control Group $(\mathrm{N}=60)$ 


\begin{tabular}{|lllllll|}
\hline Variable & \multicolumn{5}{l}{ Retinal Thickness } & \multicolumn{3}{l|}{ Retinal Volume } \\
\cline { 2 - 7 } & Control & Case & P-value & Control & Case & P-value \\
\hline Fovea & $67.8 \pm 11.4$ & $68.6 \pm 12.3$ & 0.675 & $0.053 \pm 0.009$ & $0.054 \pm 0.010$ & 0.616 \\
\hline Parafovea & $128.1 \pm 7.4$ & $129.1 \pm 9.6$ & 0.629 & $0.805 \pm 0.046$ & $0.811 \pm 0.060$ & 0.643 \\
\hline S Hemisphere & $127.7 \pm 7.7$ & $129.2 \pm 9.4$ & 0.38 & $0.401 \pm 0.024$ & $0.406 \pm 0.030$ & 0.367 \\
\hline I Hemisphere & $128.5 \pm 7.9$ & $128.9 \pm 10.2$ & 0.828 & $0.403 \pm 0.026$ & $0.405 \pm 0.032$ & 0.721 \\
\hline Temporal & $119.5 \pm 8.3$ & $120.2 \pm 9.3$ & 0.595 & $0.188 \pm 0.013$ & $0.189 \pm 0.015$ & 0.609 \\
\hline Superior & $131.0 \pm 8.1$ & $131.9 \pm 9.8$ & 0.626 & $0.206 \pm 0.013$ & $0.207 \pm 0.015$ & 0.599 \\
\hline Nasal & $130.4 \pm 8.8$ & $133.5 \pm 11.4$ & 0.191 & $0.205 \pm 0.014$ & $0.210 \pm 0.018$ & 0.193 \\
\hline Inferior & $131.4 \pm 8.4$ & $130.6 \pm 11.0$ & 0.946 & $0.206 \pm 0.013$ & $0.205 \pm 0.017$ & 0.981 \\
\hline Perifovea & $112.7 \pm 5.3$ & $113.9 \pm 8.1$ & 0.2 & $1.415 \pm 0.067$ & $1.430 \pm 0.103$ & 0.193 \\
\hline S Hemisphere & $112.8 \pm 5.6$ & $114.3 \pm 8.4$ & 0.195 & $0.709 \pm 0.035$ & $0.718 \pm 0.053$ & 0.182 \\
\hline I Hemisphere & $112.4 \pm 5.5$ & $113.3 \pm 8.3$ & 0.279 & $0.707 \pm 0.035$ & $0.712 \pm 0.052$ & 0.309 \\
\hline Temporal & $106.3 \pm 6.3$ & $107.4 \pm 7.5$ & 0.268 & $0.334 \pm 0.020$ & $0.337 \pm 0.024$ & 0.313 \\
\hline Superior & $112.0 \pm 5.8$ & $112.8 \pm 8.6$ & 0.379 & $0.352 \pm 0.018$ & $0.354 \pm 0.027$ & 0.404 \\
\hline Nasal & $121.7 \pm 8.1$ & $124.8 \pm 9.9$ & 0.133 & $0.383 \pm 0.026$ & $0.392 \pm 0.031$ & 0.127 \\
\hline Inferior & $110.0 \pm 5.4$ & $110.4 \pm 8.6$ & 0.49 & $0.346 \pm 0.017$ & $0.346 \pm 0.027$ & 0.521 \\
\hline
\end{tabular}

Notes: The unit of measurement thicknesses and volumes were $\mu \mathrm{m}$ and $\mathrm{mm}^{3}$, respectively. Values represented as mean \pm SD and analysis made by Mann-Whitney $U$ test.

Abbreviations: S, superior; I, inferior

Table 3: Comparison of the Outer Retinal Thickness between the COVID-19 Patients ( $N=25)$ and Control Group $(\mathrm{N}=60)$ 


\begin{tabular}{|lllllll|}
\hline Variable & \multicolumn{5}{l}{ Retinal Thickness } & \multicolumn{3}{l|}{ Retinal Volume } \\
\cline { 2 - 7 } & Control & Case & P-value & Control & Case & P-value \\
\hline Fovea & $177.9 \pm 11.3$ & $179.3 \pm 11.3$ & 0.71 & $0.140 \pm 0.009$ & $0.141 \pm 0.009$ & 0.717 \\
\hline Parafovea & $180.7 \pm 8.7$ & $181.9 \pm 10.5$ & 0.559 & $1.136 \pm 0.054$ & $1.143 \pm 0.066$ & 0.589 \\
\hline S Hemisphere & $181.0 \pm 9.2$ & $182.8 \pm 10.9$ & 0.566 & $0.569 \pm 0.029$ & $0.574 \pm 0.034$ & 0.524 \\
\hline I Hemisphere & $180.5 \pm 8.9$ & $181.0 \pm 10.6$ & 0.728 & $0.567 \pm 0.028$ & $0.568 \pm 0.033$ & 0.78 \\
\hline Temporal & $179.9 \pm 10.0$ & $183.6 \pm 13.1$ & 0.313 & $0.283 \pm 0.016$ & $0.288 \pm 0.021$ & 0.339 \\
\hline Superior & $181.5 \pm 9.2$ & $183.0 \pm 11.0$ & 0.54 & $0.285 \pm 0.014$ & $0.288 \pm 0.017$ & 0.537 \\
\hline Nasal & $181.2 \pm 10.9$ & $180.6 \pm 10.2$ & 0.761 & $0.285 \pm 0.017$ & $0.284 \pm 0.016$ & 0.75 \\
\hline Inferior & $180.3 \pm 8.9$ & $180.4 \pm 11.2$ & 0.981 & $0.283 \pm 0.014$ & $0.283 \pm 0.017$ & 0.996 \\
\hline Perifovea & $170.1 \pm 6.7$ & $171.6 \pm 8.9$ & 0.175 & $2.137 \pm 0.085$ & $2.156 \pm 0.111$ & 0.168 \\
\hline S Hemisphere & $171.8 \pm 7.3$ & $173.6 \pm 8.8$ & 0.143 & $1.079 \pm 0.046$ & $1.091 \pm 0.056$ & 0.14 \\
\hline I Hemisphere & $168.4 \pm 7.0$ & $169.4 \pm 9.3$ & 0.377 & $1.057 \pm 0.044$ & $1.065 \pm 0.058$ & 0.328 \\
\hline Temporal & $165.5 \pm 6.9$ & $168.8 \pm 9.4$ & 0.078 & $0.518 \pm 0.023$ & $0.530 \pm 0.030$ & 0.054 \\
\hline Superior & $172.3 \pm 7.7$ & $174.9 \pm 9.7$ & 0.08 & $0.541 \pm 0.024$ & $0.550 \pm 0.031$ & 0.073 \\
\hline Nasal & $176.1 \pm 10.5$ & $174.9 \pm 9.4$ & 0.881 & $0.553 \pm 0.033$ & $0.549 \pm 0.030$ & 0.9 \\
\hline Inferior & $166.3 \pm 7.2$ & $167.6 \pm 9.9$ & 0.372 & $0.522 \pm 0.023$ & $0.527 \pm 0.031$ & 0.367 \\
\hline
\end{tabular}

Notes: The unit of measurement thicknesses and volumes were $\mu \mathrm{m}$ and $\mathrm{mm}^{3}$, respectively. Values represented as mean \pm SD and analysis made by Mann-Whitney $U$ test.

Abbreviations: S, superior; I, inferior

\section{Discussion}

In the present study, we used SD-OCT to compare inner-, outer-, and full- retinal thickness and volume of the recovered COVID-19 patients with healthy control subjects. We found unremarkable incremental patterns in macular retinal thickness and volume in patients with a history of COVID-19. Although we failed to reach statistical significance, it seems that there is a trend toward retinal thickening in almost all sectors and different retinal layers of patients with COVID-19.

Recently, Marinho and colleagues reported hyperreflective bands in inner retinal layers in patients recovered from COVID-19 (4). Beside SD-OCT findings, they have also reported fine Cotton-Wool Spots (CWS) and microhemorrhages along the retinal arcades in four patients, suggesting an inflammatory or ischemic process. However, they did not report the detailed quantitative measures of the retinal thickness 
parameters. Moreover, they had no control group and their sample size was limited. In a later editorial on their correspondence, Vavvas et al. raised some concerns on the interpretation of the OCT and fundus findings in COVID-19 patients (5). They suggested that as CWS were very subtle and could be due to some comorbid conditions, the results provided by Marinho et al. should be interpreted with caution. In this study, we reported the detailed SD-OCT quantitative data of the retinal thickness in patients with a recent history of COVID-19 and compared our data with a healthy control group. Interestingly, we observed unremarkable changes in retinal thickness which may be in line with the theory suggested by Vavvas et al (5).

SARS-CoV-2 replication will be initiated after binding to epithelial cells in the nasopharynx and the nasal cavity. The virus propagates and migrates down the respiratory tract and triggers an innate immune response. ACE2 has been found as the main receptor for SARS-CoV-2 (16). ACE2 is an enzyme placed in cell membranes of type II alveolar cells of the lung, enterocytes of the small intestine, arterial and venous endothelial cells, and arterial smooth muscle cells in most organs (17). ACE2 counterbalances the activity of the ACE by reducing the amount of angiotensin-II and increasing angiotensin (1-7) (18). ACE2 has been found in human, rodent, and porcine retina. Moreover, ACE has been reported to present in the choroid and different cell types of the retina, including Müller cells, ganglion cells, retinal vascular endothelial cells, and photoreceptor cells (3). Hence, retina and choroid are well anticipated to be potential targets of SARS-CoV-2 infection (19).

In addition, subtle increasing in the thickness of retinal layers may be related to the minimal extra-cellular or intra-cellular fluid accumulation. Vinores et al. performed an experimental study on murine coronavirus infection in susceptible mice (BALB/C) and found that blood-retinal barrier breakdown occurs in the early phase of infection, which coincide with the onset of inflammation and indicates that blood-retinal barrier breakdown is primarily due to inflammation rather than to retinal cell destruction (20). On the other hand, apoptosis plays an important role in many ocular pathologies, such as glaucoma, retinitis pigmentosa, and diabetic retinopathy (21). Wang et al. showed that murine coronavirus induced a biphasic retinal disease in adult mice (22). The early phase was associated with inflammation, including retinal vasculitis and viral replication and the late phase was associated with retinal degeneration. Based on these pieces of evidence, we hypothesize that retinal thickening in COVID-19 patients may happen in two phases: an initial inflammatory phase with increased vascular permeability followed by a late apoptotic phase with infiltration of the retina by inflammatory cells. Although there is no direct evidence which associate the virus with retinal changes, as the virus has been found in retinal autopsy samples and

Our study had some limitations. First, we could not obtain OCT images during the acute phase of the disease. Second, our study had a relatively small sample size which might be the reason for insignificance findings. Third, since the control individuals were selected retrospectively from a large sample of an eligible cohort, selection bias might have occurred during the identification of the control arm. However, the mean thickness measures in our control group showed negligible difference with the previously published study in Iran which might be due to different measuring devices and sample 
ethnicity (23). A larger-scale study during the acute phase of the disease, followed by repeated exams at fixed intervals, would provide valuable information about the impact of COVID-19 on the retina.

\section{Conclusion}

This study shows that the patients with COVID-19 developed unremarkable thickening, mainly in parafoveal and perifoveal temporal and superior quadrants of the macula. The findings of the current study, either primarily or secondary to other causes, denote the retina could be involved in the patients with COVID-19 and highlight the necessity of comprehensive ophthalmic examinations in these patients.

\section{Abbreviations}

SD-OCT: spectral-domain optical coherence tomography (

COVID-19: Coronavirus Disease 2019

SARS-CoV-2: Severe Acute Respiratory Syndrome Corona Virus 2

RT-PCR: reverse transcription-polymerase chain reaction

CNS: central nervous system

ACE: Angiotensin-Converting Enzyme

ILM: Internal Limiting Membrane

BRM: Bruch membrane

IPL: Inner Plexiform Layer

INL: Inner Nuclear Layer

\section{Declarations}

Ethics approval and consent to participate: This report was approved by the Regional Ethic Committee at Mashhad University of Medical Sciences (IR.MUMS.REC.1399.104), to report ocular manifestations in COVID-19 patients.

Consent for publication: As our study does not contain data from any individual person, so consent for publication is not applicable

Availability of data and materials: The datasets used and/or analyzed during the current study are available from the corresponding author on reasonable request.

Competing interests: The authors declare that they have no competing interests 
Funding: This study was supported by an unrestricted institutional grant of Vice Chancellor of Research of Mashhad University of Medical Sciences (Grant Number: 990069).

Authors' contributions: MoA and FT contributed equally in the study process and preparing this report. All the authors contributed significantly to this report, and all authors agree to be accountable for all aspects of the work. All authors read and approved the final manuscript.

Acknowledgements: The authors would like to thank Mr. Hojat Salmani for his contribution in data entering process. It is a pleasure for us to also acknowledge the kind supports of Capt. Hasan Ghasemi.

\section{References}

1. Zhou Z, Kang H, Li S, Zhao X. Understanding the neurotropic characteristics of SARS-CoV-2: from neurological manifestations of COVID-19 to potential neurotropic mechanisms. J Neurol. 2020:1-6.

2. Liu M-Y, Zheng B, Zhang Y, Li J-P. Role and mechanism of angiotensin-converting enzyme 2 in acute lung injury in coronavirus disease 2019. Chronic Dis Transl Med. 2020;6(2):98-105.

3. Choudhary R, Kapoor MS, Singh A, Bodakhe SH. Therapeutic targets of renin-angiotensin system in ocular disorders. Journal of current ophthalmology. 2017;29(1):7-16.

4. Marinho PM, Marcos AAA, Romano AC, Nascimento $H$, Belfort R, Jr. Retinal findings in patients with COVID-19. Lancet. 2020;395(10237):1610-.

5. Vavvas DG, Sarraf D, Sadda SR, Eliott D, Ehlers JP, Waheed NK, et al. Concerns about the interpretation of OCT and fundus findings in COVID-19 patients in recent Lancet publication. Eye (Lond). 2020:1-2.

6. Casagrande M, Fitzek A, Püschel K, Aleshcheva G, Schultheiss HP, Berneking L, et al. Detection of SARS-CoV-2 in Human Retinal Biopsies of Deceased COVID-19 Patients. Ocular immunology and inflammation. 2020:1-5.

7. Pons-Escoda A, Naval-Baudín P, Majós C, Camins A, Cardona P, Cos M, et al. Neurologic Involvement in COVID-19: Cause or Coincidence? A Neuroimaging Perspective. AJNR American journal of neuroradiology. 2020.

8. Gklinos P. Neurological manifestations of COVID-19: a review of what we know so far. J Neurol. 2020:1-5.

9. Gupta S, Zivadinov R, Ramanathan M, Weinstock-Guttman B. Optical coherence tomography and neurodegeneration: are eyes the windows to the brain? Expert Rev Neurother. 2016;16(7):765-75.

10. Knier B, Berthele A, Buck D, Schmidt P, Zimmer C, Muhlau M, et al. Optical coherence tomography indicates disease activity prior to clinical onset of central nervous system demyelination. Mult Scler. 2016;22(7):893-900.

11. Abrishami M, Tohidinezhad F, Daneshvar R, Omidtabrizi A, Amini M, Sedaghat A, et al. Ocular Manifestations of Hospitalized Patients with COVID-19 in Northeast of Iran. Ocular immunology and inflammation. 2020:1-6. 
12. Seah I, Agrawal R. Can the Coronavirus Disease 2019 (COVID-19) Affect the Eyes? A Review of Coronaviruses and Ocular Implications in Humans and Animals. Ocul Immunol Inflamm. 2020;28(3):391-5.

13. Seah IYJ, Anderson DE, Kang AEZ, Wang L, Rao P, Young BE, et al. Assessing Viral Shedding and Infectivity of Tears in Coronavirus Disease 2019 (COVID-19) Patients. Ophthalmology. 2020.

14. Mungmungpuntipantip R, Wiwanitkit V. Ocular manifestation, eye protection, and COVID-19. Graefes Arch Clin Exp Ophthalmol. 2020;258(6):1339.

15. Tohidinezhad F, Khorsand A, Zakavi SR, et al. The burden and predisposing factors of noncommunicable diseases in Mashhad University of Medical Sciences personnel: a prospective 15-year organizational cohort study protocol and baseline assessment. BMC Public Health. 2020;20(1):1637. Published 2020 Nov 2.

16. Wan Y, Shang J, Graham R, Baric RS, Li F. Receptor Recognition by the Novel Coronavirus from Wuhan: an Analysis Based on Decade-Long Structural Studies of SARS Coronavirus. Journal of virology. 2020;94(7).

17. Hamming I, Timens W, Bulthuis MLC, Lely AT, Navis GJ, van Goor H. Tissue distribution of ACE2 protein, the functional receptor for SARS coronavirus. A first step in understanding SARS pathogenesis. J Pathol. 2004;203(2):631-7.

18. Chamsi-Pasha MA, Shao Z, Tang WH. Angiotensin-converting enzyme 2 as a therapeutic target for heart failure. Current heart failure reports. 2014;11(1):58-63.

19. Seah I, Agrawal R. Can the Coronavirus Disease 2019 (COVID-19) Affect the Eyes? A Review of Coronaviruses and Ocular Implications in Humans and Animals. Ocular immunology and inflammation. 2020;28(3):391-5.

20. Vinores SA, Wang Y, Vinores MA, Derevjanik NL, Shi A, Klein DA, et al. Blood-retinal barrier breakdown in experimental coronavirus retinopathy: association with viral antigen, inflammation, and VEGF in sensitive and resistant strains. Journal of neuroimmunology. 2001;119(2):175-82.

21. Papermaster DS, Windle J. Death at an early age. Apoptosis in inherited retinal degenerations. Investigative ophthalmology \& visual science. 1995;36(6):977-83.

22. Wang Y, Detrick B, Yu ZX, Zhang J, Chesky L, Hooks JJ. The role of apoptosis within the retina of coronavirus-infected mice. Investigative ophthalmology \& visual science. 2000;41(10):3011-8.

23. Hashemi H, Khabazkhoob M, Yekta A, Emamian MH, Nabovati P, Fotouhi A. The Distribution of Macular Thickness and Its Determinants in a Healthy Population. Ophthalmic epidemiology. 2017;24(5):323-31. 